\title{
Intravesical RNA activation-a new treatment concept
}

US researchers have demonstrated the therapeutic potential of delivering small activating RNAs (saRNAs) directly into the bladder to treat cancer. The use of RNA interference to block the production of cancer-related proteins has been the topic of much research over the last decade. RNA activation, on the other hand, is a relatively new concept. "To our best knowledge, our study represents the largest cohort of animals ever used to evaluate saRNA-based therapeutics for treating bladder cancer in a preclinical setting," says Long-Cheng Li, corresponding author.

Several saRNAs have been identified that induce transcription of target proteins; for example, dsP21-322 stimulates production of the tumour suppressor $\mathrm{p} 21$. Li and his team were aware that $\mathrm{p} 21$ overexpression might inhibit tumour growth based on their previous experiments in cultured bladder cells. Keen to test the function of dsP21322 in vivo, they first had to modify the saRNA to improve its stability in the bladder. Urine is rich in ribonucleases that can rapidly degrade unmodified dsP21-322, but the investigators found that 2'-fluoromodification extended its half-life. A further concern was that the protective glycosaminoglycan (GAG) layer, which lines the bladder, might prevent uptake of saRNA into target tissue. For this reason, $\mathrm{Li}$ and colleagues used a lipid nanoparticle delivery system. Bioavailability and pharmacokinetic experiments in normal mouse bladder revealed that the saRNA nanoparticles were indeed able to penetrate the GAG layer and were internalized by urothelial cells.

\section{$\mathbf{4}$...mice that had received active nanoparticles demonstrated significantly prolonged survival... 77}

Finally, researchers evaluated the efficacy of the saRNA nanoparticles in mice with orthotopic bladder cancer. After 8 weeks of intravesical treatment, mice that had received active nanoparticles demonstrated significantly prolonged survival (median 45 days; $n=10$ ) compared to those that received control nanoparticles (median 13 days; $n=10$ ) or saline injections (median 9 days; $n=9$ ). Strikingly, four of the five treated mice that survived demonstrated tumour regression and three eventually became tumourfree. Immunohistochemical analysis of harvested bladders revealed increased levels of both p21 and apoptosis.

$\mathrm{Li}$ and colleagues are now hoping to secure funding for a clinical trial of this saRNA drug. "We believe that intravesical RNA therapy is a very promising therapeutic modality for bladder cancer," asserts $\mathrm{Li}$, "especially when used as adjuvant therapy after transurethral resection or for carcinoma in situ, a highly aggressive form of bladder cancer."

Sarah Payton

Original article Kang, M. R. et al. Intravesical delivery of small activating RNA formulated into lipid nanoparticles inhibits orthotopic bladder tumor growth. Cancer Res. doi:10.1158/0008-5472.CAN-12-1871 